\title{
Virus-induced gene silencing in Nicotiana benthamiana triggered by heterologous gene sequences from Viola philippica
}

\author{
Q.X. $\mathrm{LI}^{1 *}$, J. WANG ${ }^{1}, \mathrm{~S} . \mathrm{ZHENG}^{1}, \mathrm{~N} . \mathrm{YANG}^{1}, \mathrm{~K} . \mathrm{SUN}^{1 *}$, and C.Y. $\mathrm{HE}^{2}$ \\ Life Science College, Northwest Normal University, Anning, 730070 Lanzhou, Gansu, P.R. China ${ }^{1}$ \\ State Key Laboratory of Systematic and Evolutionary Botany, Institute of Botany, Chinese Academy of Sciences, \\ Xiangshan, 100093 Beijing, P.R. China ${ }^{2}$
}

\begin{abstract}
Virus-induced gene silencing (VIGS) is a particularly useful tool for functional genomics. In the present study, we attempted to utilize this technology to infer the function of genes from Viola philippica using a tobacco rattle virus (TRV) construct. Firstly, the phytoene desaturase gene from $V$. philippica (VpPDS) was silenced, and local leaf bleaching was observed but did not exhibit systemic effects, thereby limiting utilization of TRV-mediated gene silencing in the recipient plant. However, we observed systemic gene silencing in Nicotiana benthamiana when the VpPDS sequence was used as a trigger, thereby suggesting that heterologous gene sequences could elicit gene silencing. We then investigated the role of gene PISTILLATA from $V$. philippica $(V p P I)$ of the B-class MADS-box gene family, which regulates the identity and development of stamens and petals. Using the coding region of $V p P I$ as triggers, we determined the gene silencing efficiency of the corresponding GLOBOSA paralogs in N. benthamiana ( NbGLO1 and NbGLO2), and we observed stamen-to-carpel transformation and distorted corollas in $N$. benthamiana suggesting that $V p P I$ functioned as the $N b G L O$ gene. However, the 3'-untranslated region (3'UTR) of $V p P I$ and each 3'UTR of the $N b G L O$ genes downregulated its own corresponding gene, hardly producing floral homeotic alterations. This work provides a better understanding of gene-specific probe design for gene silencing as well as shows that heterologous sequence-triggered VIGS is an efficient way to investigate functional conservation of orthologous genes.
\end{abstract}

Additional key words: gene-specific probe, GLOBOSA, phytoene desaturase, PISTILLATA.

\section{Introduction}

Virus-induced gene silencing (VIGS) is a method that utilizes RNA interference (RNAi) to induce transient gene knockdown in plants (Dinesh-Kumar et al. 2003). Virusinduced gene silencing exploits the plant endogenous RNA-mediated defense system that targets viral RNAs for sequence-specific degradation (Robertson 2004, Becker and Lange 2010). A previous study showed that a doublestrand RNA (dsRNA) fragment (a silencing inducer) 300 $500 \mathrm{bp}$ in length with at least one stretch of $21-23$ nucleotides with $100 \%$ identity to a targeted transgene mRNA is essential to gene silencing in plants (Thomas et al. 2001). Virus-induced gene silencing is an effective technology for the analysis of gene functions because it is easy, rapid, and circumvents the need for genetic transformation of plants. Recently, VIGS has been performed in various dicot plant species including those from Solanaceae (Nicotiana spp., Solanum spp., Capsicum annuum, Lycium spp., and Physalis floridana; Kumagai et al. 1995, Ratcliff et al. 1997, 2001, Liu et al. 2002, Brigneti et al. 2004, Chung et al. 2004, Senthil-Kumar et al. 2007, Liu et al. 2014, Zhang et al. 2014a,b, Meng et al. 2016), Leguminosae (Pisum sativum and Glycine max; Constantin et al. 2004, Zhang and Ghabrial 2006,

Submitted 8 February 2018. last revision 5 July 2018, accepted 1 August 2018.

Abbreviations: AP1 - APETALA1; cDNA - complementary DNA; GLO - GLOBOSA; ORF - open reading frame; PDS - phytoene desaturase; PI - PISTILLATA; quantitative PCR - qPCR; RNAi - RNA interference; TRV - tobacco rattle virus; VIGS - virus-induced gene silencing; UTR - untranslated region.

Acknowledgements: The help of Dr. Jing Li (State Key Laboratory of Systematic and Evolutionary Botany, Institute of Botany, Chinese Academy of Sciences) in vector construction is acknowledged. This work was supported by the NSFC grant (31560066) and the CAS/SAFEA International Partner Program for Creative Research Teams of "Systematic and Evolution Botany".

* Corresponding authors: liqiaoxia8024@163.com; kunsun@163.com 
Zhang et al. 2010), Brassicaceae (Arabidopsis thaliana; Burch-Smith et al. 2006, Pflieger et al. 2008), Ranunculaceae (Aquilegia vulgaris; Gould and Kramer 2007), Malvaceae (Gossypium hirsutum; Gao et al. 2011, Tuttle et al. 2012), Rosaceae (Malus pumila; Sasaki et al. 2011), and some monocot species such as Hordeum vulgare (Hein et al. 2005, Yuan et al. 2011), Triticum aestivum (Ma et al. 2012), Zea mays (Benavente et al. 2012), and Oryza sativa (Purkayastha et al. 2010).

Viola philippica is a common species with the typical chasmogamous-cleistogamous mixed breeding system. $V$. philippica develops chasmogamous flowers under a short photoperiod, whereas an extended photoperiod induces the formation of cleistogamous flowers ( $\mathrm{Li}$ et al.

\section{Materials and methods}

Sequence cloning: Total RNA was extracted from leaves and flowers of Nicotiana benthamiana Domin and leaves of Viola philippica Cav. using a TRIzol® reagent (Tiangen, Beijing, China), and first-strand cDNA synthesis was performed using a PrimeScript RT reagent kit (TaKaRa, Dalian, China). The cDNA fragments of phytoene desaturase of $V$. philippica (VpPDS) were isolated using degenerate primers (Table 1 Suppl.), which were designed based on the conserved regions of the PDS orthologs from various plant species. The full-length cDNA was assembled by 3'- and 5'-rapid amplification of cDNA ends using gene-specific primers (Table 1 Suppl.). Universal $3^{\prime}$ and 5' PCR primers were supplied by a SMARTer ${ }^{\mathrm{TM}}$ cDNA library construction kit (Clontech, Mountain View, CA, USA). After rapid amplification of cDNA ends, the full-length cDNA was amplified by routine reverse transcription (RT) PCR using gene-specific primers (Table 1 Suppl.). The cDNA of $V p P D S$ and $V p P I$ of $V$. philippica and the cDNA of $N b G L O 1, N b G L O 2$, and NbAPl (APETALA1) of $N$. benthamiana were amplified using gene-specific or degenerate primers (Table 1 Suppl.). The Ex Taq enzyme (TaKaRa) was used in routine reverse transcription (RT)-PCR. The amplified cDNA fragments were cloned using a vector pMD19-T (TaKaRa) and sequenced by Huada (Beijing, China).

The VIGS plasmid construction: The $p T R V 1$ and $p T R V 2$ VIGS vectors (Fig. 1 $A, B$ ) were used in this study as previously described (Liu et al. 2002, Gould and Kramer 2007). The probe of each gene was amplified from the obtained cDNAs of $V p P D S, V p P I, N b G L O 1$, and $N b G L O 2$ using gene-specific primers (Table 1 Suppl.). The amplified gene fragments were cloned into the $p T R V 2$ vector using restriction enzymes $N c o$ I and BamHI. The constructs TRV2::VpPDS-ORF, TRV2::VpPI-ORF, TRV2::VPPI-3'UTR, TRV2::NbGLO1-3'UTR, and TRV2::NbGLO2-3'UTR (Fig. $1 C-G$ ) were then generated. Positive clones of the above-mentioned VIGS constructs were detected using 156 primers (located in the $p T R V 2$
2016), thereby serving as a good model for investigating the developmental evolution of dimorphic flowers. In the present study, we explored the tobacco rattle virus (TRV)mediated VIGS of genes belonging to two families in $V$. philippica. Phytoene desaturase (PDS) is an important enzyme in the carotenoid biosynthetic pathway, in which silencing leads to chlorophyll photo-oxidation (Kumagai et al. 1995), whereas PISTILLATA (PI) or GLOBOSA $(G L O)$ of the B-class of MADS-box gene family regulates the identity and development of stamens and petals (Bowman et al. 1989, Hill and Lord 1989). Our aim was to provide new insights into investigating $V$. philippica gene function in $N$. benthaminan based on heterologous sequence-triggered VIGS.

vector) and sequenced by Huada (Beijing, China). The plasmid constructs were extracted using a TIANprep mini plasmid kit (Tiangen).

Plant growth and agroinfiltration: Plasmids were introduced into Agrobacterium tumefaciens L. strain GV3101 by heat shock. The $A$. tumefaciens strain GV3101 containing $p T R V 1$ and pTRV2-derivatives (Fig. 1C-G) were plated on a yeast and beef extract (YEB) solid medium containing kanamycin $\left(50 \mu \mathrm{g} \quad \mathrm{cm}^{-3}\right)$ and rifampicin $\left(100 \mu \mathrm{g} \mathrm{cm}^{-3}\right)$ and cultivated at a temperature of $28^{\circ} \mathrm{C}$ in the dark. Two days later, a single colony was selected and inoculated into $5 \mathrm{~cm}^{3}$ of YEB liquid medium, which contained $100 \mu \mathrm{g} \mathrm{cm}^{-3}$ rifampicin and $50 \mu \mathrm{g} \mathrm{cm}^{-3}$ kanamycin and cultured overnight on a shaker (250 rpm). Then, the cultures were transferred into $250 \mathrm{~cm}^{3}$ of YEB liquid medium containing $100 \mu \mathrm{g} \mathrm{cm} \mathrm{cm}^{-3}$ rifampicin, $50 \mu \mathrm{g} \mathrm{cm}^{-3}$ kanamycin, $10 \mathrm{mM}$ 2-(N- morpholino) ethanesulfonic acid (MES), and $0.2 \mathrm{mM}$ acetosyringone and grown overnight at $28{ }^{\circ} \mathrm{C}(250 \mathrm{rpm})$. Agrobacterium cells were harvested by centrifugation at $4000 \mathrm{~g}$ for $15 \mathrm{~min}$, and the pellets were resuspended in an infiltration buffer $(10 \mathrm{mM} \mathrm{MgCl} 2,10 \mathrm{mM} \mathrm{MES}$, and $200 \mathrm{mM}$ acetosyringone) to reach an absorbance of 2.0 at a wavelength of $600 \mathrm{~nm}$. Infiltration cultures were incubated at room temperature in a triangle flask for $3 \mathrm{~h}$, and the $\mathrm{p} T R V 1$ and $\mathrm{p} T R V 2$ derivative solutions were mixed at $1: 1$ $(\mathrm{v} / \mathrm{v})$ ratios and then infiltrated into the underside of all leaves of four-leaf stage plants of $N$. benthamiana and $V$. philippica using a $1-\mathrm{cm}^{3}$ needleless syringe. The infiltrated $N$. benthamiana and $V$. philippica plants were cultured in the dark for $2 \mathrm{~d}$ and then were kept at a temperature of 22 - $25{ }^{\circ} \mathrm{C}$, a 16-h photoperiod, an irradiance of $120 \mu \mathrm{mol} \mathrm{m} \mathrm{m}^{-}$ $2 \mathrm{~s}^{-1}$, and an air humidity of $48 \%$ for phenotypic observation.

Real-time quantitative RT-PCR analysis: To quantify transcript abundances of $V p P D S, N b P D S, N b A P 1$, and B-class MADS-box genes in inoculated $V$. philippica and 
$N$. benthamiana plants, real-time quantitative (q) PCR was performed using three independent biological replicates. To reduce errors, the collection of flowers or leaves were processed at the same time and in a shortest time. Total RNA was extracted from the leaves or flowers using a TRIzol® reagent (Tiangen). RNA concentrations were measured using an ultramicrospectrophotometer (NanoDrop2000, Thermo Scientific, Massachusetts, USA), and the concentrations of all samples were adjusted to be the same. For qPCR analysis, a PrimeScript $R T$ reagent kit (TaKaRa), SYBR Premix EX Taq II (TaKaRa), and gene-specific primers were used (Table 1 Suppl.). The Vp18sRNA and Nbactin RNA genes were used as internal references for $V$. philippica and $N$. benthamiana, respectively. The amplification conditions were $95{ }^{\circ} \mathrm{C}$ for $30 \mathrm{~s}$ for one cycle followed by 40 cycles of $95{ }^{\circ} \mathrm{C}$ for $5 \mathrm{~s}$ and $60{ }^{\circ} \mathrm{C}$ for $30 \mathrm{~s}$. Three independent biological samples were used. Expressions were calculated according to Livak and Schmittgen (2001).

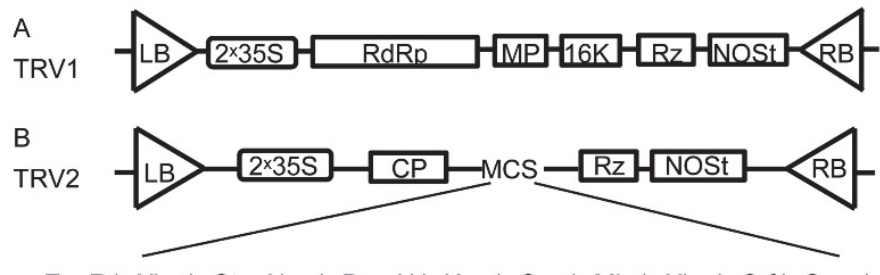

EcoR1, Xba1 ,Stu, Nco1, BamH1, Kpn1, Sac1, Mlu1, Xho1, Srf1, Sma1

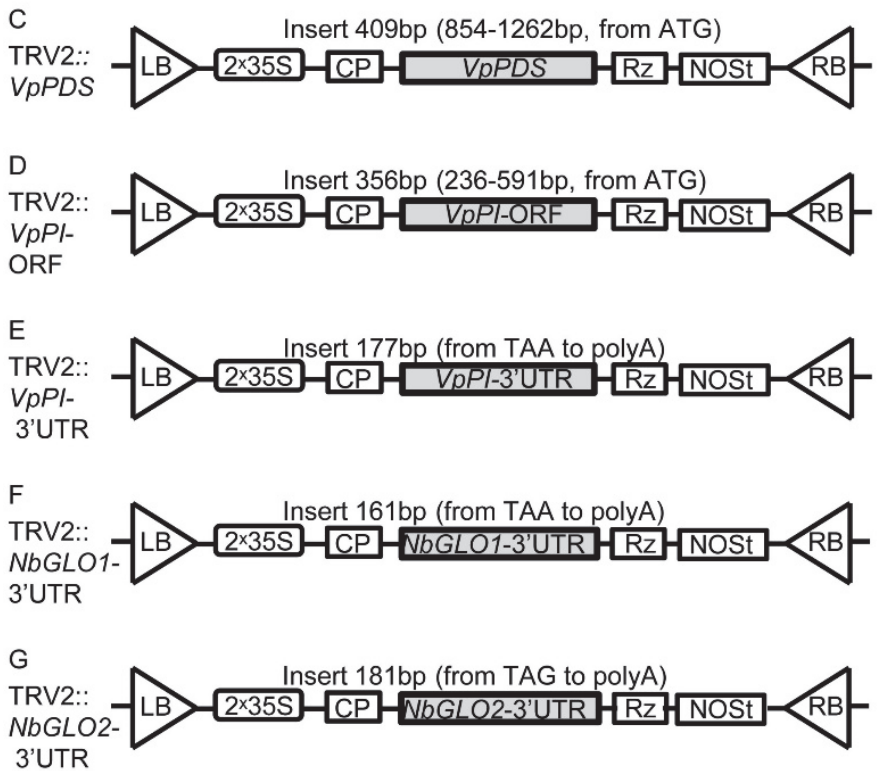

Fig. 1. Virus induced gene silencing (VIGS) constructs generated in this study. A A scheme of the tobacco rattle virus 1 (TRV1) vector. $B$ A scheme of the $T R V 2$ vector. $C$ to $G$ - Schemes of the $T R V 2$-based gene-specific VIGS constructs. The gene-specific primer positions are indicated, and the sequences are presented in Table 1 Suppl. LB - left border; RB - right border; RdRp - RNA-dependent RNA polymerase; MP - movement protein; $16 \mathrm{~K}$ - $16 \mathrm{kd}$ protein; $\mathrm{Rz}$ - self-cleaving ribozyme; NOSt - NOS terminator; CP - coat protein; MCS - multiple cloning site; ORF - open reading frame; 3'UTR - 3'-untranslated regions. The schemes of the TRV1 and TRV2 vectors were modified from the previous investigations (Liu et al. 2002, Gao et al. 2011).

Morphological observations: Flower bud morphology was assessed under a stereomicroscope (Olympus SZ61, Tokyo, Japan). Ten flower buds from each plant were observed, and 28 plants for each gene silencing were used for statistical analysis. Representative flower images were taken using a camera linked to the stereo-microscope. Gene silencing frequency was defined as the ratio of the number of plants showing a silencing phenotype (bleaching or abnormal flower buds) and the total number of plants infiltrated (Senthil-Kumar et al. 2007).
Sequencing analysis: The nucleotide sequences of the genes were aligned using CLUSTALW as implemented in MegAlign (http://www.dnastar.com), and sequence identities were calculated. The multiple sequence alignments were saved as GCG pileup files and exported using GeneDoc (http://genedoc.software. informer.com) with manual adjustments, and gaps were introduced for proper alignment. Neighbor-joining phylogenetic trees of protein sequences were constructed using MEGA5 (Tamura et al. 2011). Bootstrap values were based on 1000 replicates. 
Statistics: Means \pm standard deviations (SDs) were calculated from three independent biological replicates. Significance differences and the related correlations were analyzed in SPASS13.0.

Data availability: The sequences reported in this study have been deposited in GenBank (http:/www.ncbi. nlm.nih.gov) as accession numbers KU318324 (VpPI), MF156690 (VpPDS), MF156691 [NbGLO1, open reading frame (ORF)], MF156692 (NbGLO2, ORF), HQ005417 [NbGLO1, 3'-untranslated region (3'UTR)], HQ005418 (NbGLO2, 3'UTR), and MG637440 (NbAPl).

\section{Results}

To apply VIGS in $V$. philippica, we firstly tried to silence $V p P D S$. The leaves that were inoculated with A. tumefaciens carrying TRV1 and TRV2::VpPDS exhibited photobleaching, whereas the newly developed leaves were as green as the wild-type leaves (Table 2 Suppl. and Fig. 1 Suppl.). Furthermore, VpPDS expression was significantly reduced in the photobleached leaves (N1 - N4) correlating with the visual observations on the area of white patches, whereas no change was observed in the young leaves of $V$. philippica and the non-photobleached ones, which were designated as N0 leaves (Table 2 Suppl. and Fig. 1 Suppl.). These results indicate that TRV-mediated local gene silencing was possible in Viola, although no efficient and systemic gene silencing occurred.

In parallel, we used the $V$. philippica sequence as the heterologous gene sequence for VIGS in N. benthamiana. $N$. benthamiana was inoculated with $T R V 2: \because V p P D S$ and

one month later, $100 \%$ of the plants exhibited photobleaching symptoms (Fig. $2 A, B$ and Table 2 Suppl.). Systemic gene silencing and the consequent phenotypic variations were observed in the newly developed leaves of $N$. benthamiana (Fig. 2B), and no floral homeotic changes were observed. Real-time qPCR analyses indicate a significant reduction in endogenous $P D S$ expression in the visually categorized N1 - N4 photobleached leaves of $N$. benthamiana (Fig. 2C). Thus, the heterologous sequence of $V$. philippica could knock down the corresponding ortholog or closely related homologs in N. benthamiana.

Based on this finding, we investigated the function of the VPPI gene (Li et al. 2016). Phylogenetic reconstruction in combination with sequence identity suggested that $V p P I$ is the putative ortholog of $N b G L O 2$ in $N$. benthamiana (Fig. 2 Suppl.).
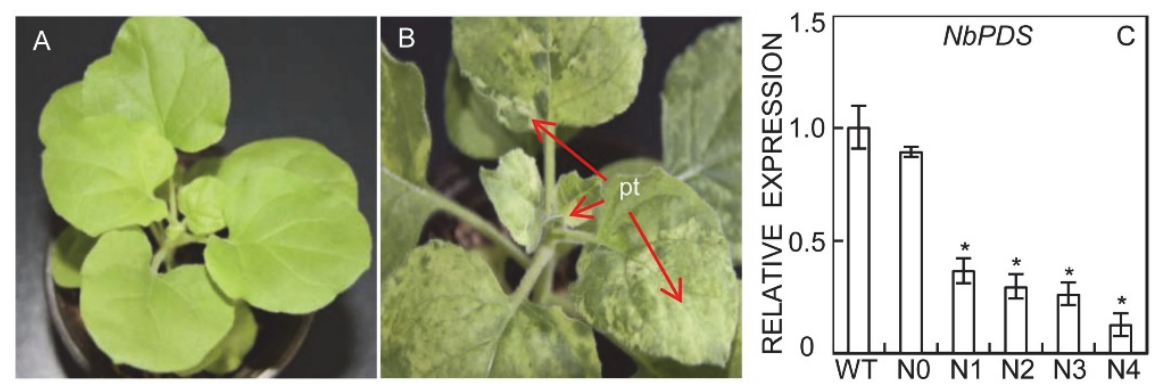

Fig. 2. Gene silencing in Nicotiana benthamiana using $V p P D S$ as a trigger sequence. $A$ - Wild-type. $B$ - Silencing endogenous $N b P D S$ genes in $N$. benthamiana by inoculating with Agrobacterium tumefaciens containing TRV2::VpPDS. The red arrows indicate the white patches on the leaves (pt - photobleaching). $C$ - Relative expression of $N b P D S$ in the leaves of $N$. benthamiana; WT - wild-type leaves; N0 - WT-like leaves with no photobleaching; leaves showing an increased severity of photobleaching were visually categorized to N1 - N4 according to the area of white patches. Means \pm SDs, $n=3$. * indicate significant differences at $P \leq 0.01$ relative to the WT. The leaves for quantitative PCR analysis were collected about $35 \mathrm{~d}$ post-inoculation.

To silence the $N b G L O$ genes, we firstly inoculated the leaves of $N$. benthamiana with TRV2::VpPI-ORF (Fig. 1D). Approximately $54 \%$ of the inoculated plants generated flowers with abnormal morphological structures, particularly the stamens and petals (Fig. 3A-J and Table 2 Suppl.). Compared to the wild-type flowers (Fig. 3A), stamen-to-pistil (ovule) transformation was mainly observed in the TRV2::VpPI-ORF plants, and the number of transformed organs varied from one to five (Fig. 3B-J). The TRV2::VpPI-ORF plants depicted five degrees of variation, which we designated as N0 - N4. The
N0 flowers resembled the wild-type flowers. The N1 flowers harbored stamens with microspores, which were transformed into ovules, and no stamen adnation to the corolla tube was observed, thereby generating free stamens (Fig. 3B,C). The tops of the stamens in the N2 flowers generated stigmas and stamens changed into ovules (Fig. 3D,E). Nonetheless, the petals did not show any significant changes. In the N3 flowers, some stamens were completely transformed into pistils (Fig. $3 F, G$ ), whereas the N4 flowers displayed wrinkled and distorted petals (Fig. 3H), and the stamens were completely 
transformed into pistils (Fig. $3 I, J$ ).

To elucidate the mechanism underlying the observed floral phenotypic variations, we investigated the

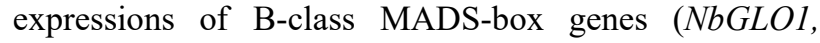

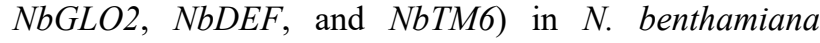
flowers that displayed different degrees of phenotypic variations. We also assessed the expression of APETALA1 $(N b A P 1)$, which belongs to the A-class MADS-box gene family, a close homolog of B-class MADS-box genes. The expressions of all B-class MADS-box genes, particularly $N b G L O 2$, which shared the highest identity with $V p P I$ (Fig. 2 Suppl.) were significantly reduced in the abnormal flowers of $N$. benthamiana compared to the wild-type flowers (Fig. $3 K$ ). However, the variation in $N b A P 1$ expression was antithetical to that of the B-class MADSbox genes in $N$. benthamiana suggesting the specificity of gene silencing. Upregulation of $N b A P 1$ by downregulating $\mathrm{NbGLO2}$ was in line with the observation in Arabidopsis, where $A P 1$ transcription increase when the expressions of B-class MADS-box genes decrease (Sundström et al. 2006). Moreover, we observed systemic silencing all B-class MADS-box genes (Fig. $3 K$ and Table 3 Suppl.). A correlation between the extent of floral variation and the reduction in the expression of these genes was observed (NbGLO1: $r=0.934, P<0.01 ; N b G L O 2: r=0.968$, $P<0.01 ; N b D E F: r=0.951, P<0.01 ;$ NbTM6: $r=0.805$, $P<0.01)$. The N4 flowers that exhibited wrinkled petals and five stamens that were fully transformed to pistils showed the most significant reductions in gene expression, whereas the N0 flowers with petals and stamens that remained unchanged depicted the smallest decreases in gene expression (Fig. 3K). The systemic silencing effect of all B-class MADS-box genes might be due to the high sequence identity between the trigger sequence $V p P I$ and the target sequence $N b G L O 2$ and its homologs (Table 1 and Table 3 Suppl.).

To exclude this possibility, we inoculated $N$. benthamiana plants with TRV2::VpPI-3'UTR (Fig. 1E). In this case, no gene silencing effect was expected because the 3'UTR is relatively gene-specific. However, the results indicate that $21 \%$ of the VIGS plants had flowers with abnormal morphological structures, particularly the petals (Fig. 4 and Table 2 Suppl.). Compared with the wild-type flowers (Fig. 4A), N0 flowers, which resembled the wildtype flowers of the TRV2::VpPI-3'UTR plants, were observed although floral variations including unhealed
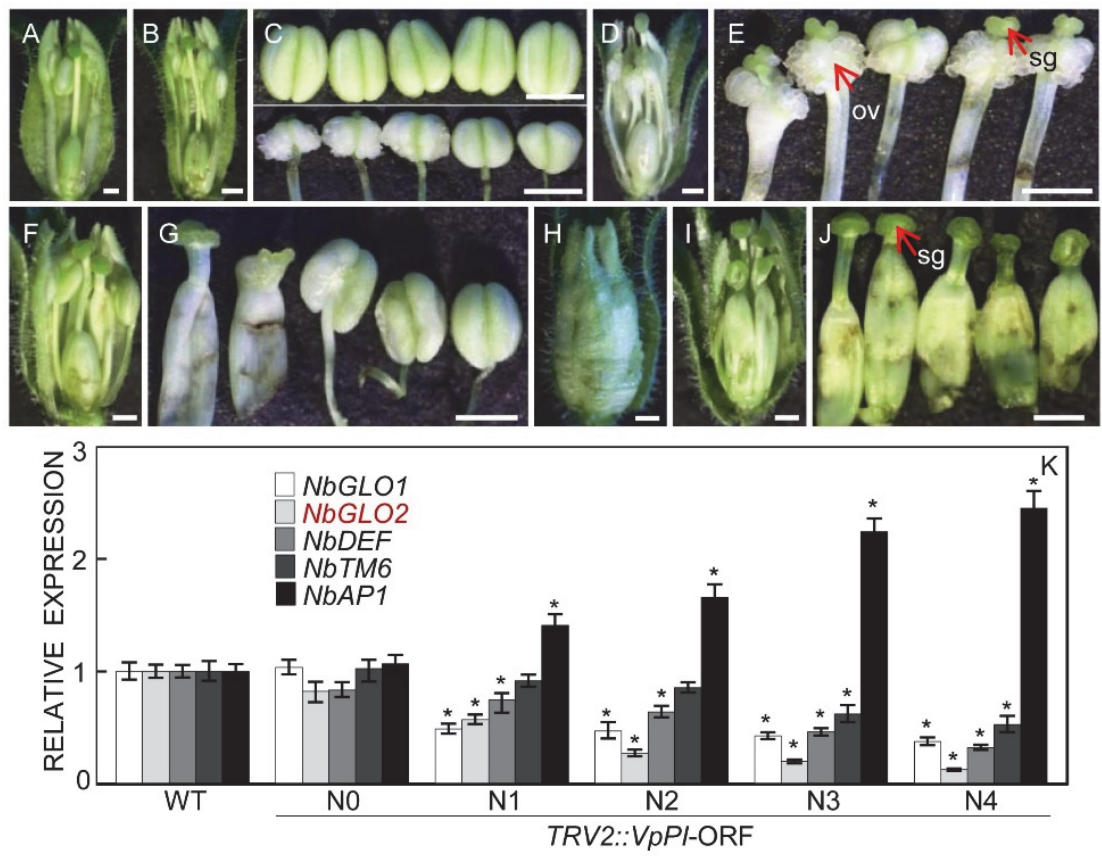

Fig. 3. Silencing Globosa $(G L O)$ genes using the open reading frame (ORF) fragment of the $V p P I$ gene. $A$ - A wild-type flower. $B$ - A VIGS flower displaying anthers that have undergone dehiscence and ovules. $C$-Stamens from a wild-type flower (the upper part) and from a virus-induced gene silencing (VIGS) flower (a lower part). D - A VIGS flower without stamen adnation to the corolla tube, thereby resulting in free stamens. $E$ - Five stamens had stigma-like structures, and anthers were transformed into ovules. $F$ - A VIGS flower displaying anthers, which were transformed into pistils. $G$ - The transformed pistils from the stamens in $F$. $H$ - A VIGS flower showing a crumpled corolla tube. $I$ - A VIGS flower with a crumpled corolla tube in $H$ showing that five stamens were all transformed into pistils. $J$ - The transformed pistils from stamens from the VIGS flower in $H$ and $I$. ov - ovules; sg - stigma-like structure. $K$ - Relative expressions of B-class genes and NbAP1 in VIGS flowers. WT - wild-type flower, N0 - VIGS flowers resembling WT, $\mathrm{N} 1$ - flowers with one to three anthers that transformed into ovules, N2 - flowers with five stamens that have stigma-like structures and anthers transformed into ovules, N3 - flowers with one to three stamens that transformed into pistils, and N4 - flowers with five stamens that transformed into pistils and wrinkled petals. Means \pm SDs, $n=3, *-$ significant differences at $P \leq 0.01$ relative to the WT. The flowers for quantitative PCR analysis were collected about $60 \mathrm{~d}$ post-inoculation. 
petal tubes (N1 flowers; Fig. 4B), and distorted and wrinkled petals were observed (N2 and N3, Fig. 4C,D). Besides NbTM6, the expressions of three other genes, particularly $\mathrm{NbGLO2}$, were significantly reduced in these flowers (Fig. $4 E$ and Table 3 Suppl.). The auto- and crossregulatory circuit of obligate heterodimers between $B$ class MADS-box genes was required for the specification of petal and stamen identity in angiosperms (Zahn et al. 2005, Zhang et al. 2014a). Thus, the reciprocal regulations among B-class MADS-box genes could account for the reduction of expressions of $N b G L O 1, N b D E F$, and NbTM6 when $N b G L O 2$ was silenced in $N$. benthamiana by the VpPI-3'UTR probe (Geuten and Irish 2010, Zhang et al. 2014a). Furthermore, the expression of $N b A P 1$ increased in the abnormal flowers of $N$. benthamiana compared with the wild-type flowers, whereas the expressions of B-class MADS-box genes in the TRV2::VpPDS flowers were similar to the wild-type flowers (Fig. $4 E$ ).

These results demonstrate that the heterologous gene sequence of $V p P I$, either the ORF or the 3'UTR, could specifically knock down its putative ortholog $N b G L O 2$ in $N$. benthamiana, thereby leading to floral alterations. Therefore, $V p P I$ in Viola played a role similar to that of its ortholog NbGLO2 in Nicotiana in controling petal and stamen identity and development.
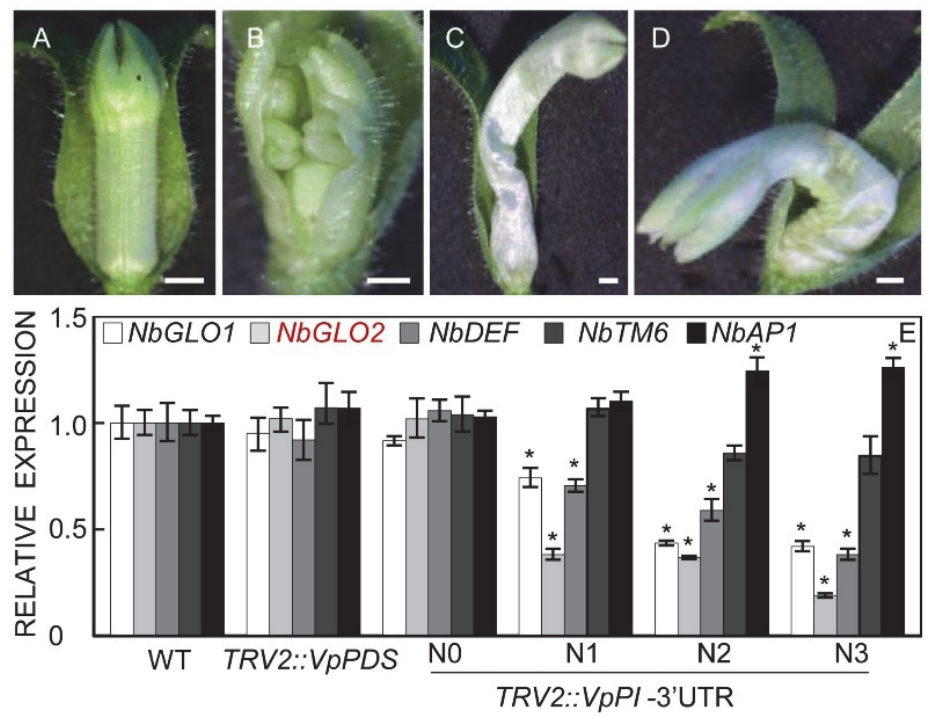

Fig. 4. Silencing $N b G L O$ by the 3'UTR sequence of the $V p P I$ gene. $A$ - A wild-type flower. $B$ - A flower of a TRV2::VpPI-3'UTRinoculated plant; an unhealed petal tube. $C$ - A flower of a TRV2:VVPI-3'UTR-inoculated plant. The petals became slightly wrinkled. $D$ - A flower of a TRV2::VPPI-3'UTR-inoculated plant. The petals became severely wrinkled. $E$ - Relative expressions of B-class genes and $N b A P 1$ in virus-induced gene silencing (VIGS) flowers. WT - wild-type flowers; TRV2::VpPDS - flowers of $T R V 2: \because V p P D S$ VIGS plants; N0 to N3 - flowers of TRV2::VpPI-3'UTR VIGS plants; N0 - flowers that resembled WT; N1 - flowers with unhealed petal tubes; N2 - flowers with slightly distorted corolla tubes; N3 - flowers with severely crumpled corolla tubes. Means \pm SDs, $n=3$, * - significant differences at $P \leq 0.01$ relative to WT. The flowers were collected about $60 \mathrm{~d}$ post-inoculation.

An apparent weak gene silencing effect was observed when the 3'UTR of $V p P I$ was employed as a trigger sequence. To objectively evaluate this effect, we inoculated the leaves of $N$. benthamiana with TRV2::NbGLO1-3'UTR (Fig. 1F) and TRV2::NbGLO23'UTR (Fig. $1 G$ ). No mutated flowers were observed in the TRV2::NbGLO1-3'UTR plants, whereas $32 \%$ of the TRV2::NbGLO2-3'UTR plants produced abnormal flowers (Fig. 5 and Table 2 Suppl.). Unlike the flowers from the wild-type and the TRV2::NbGLO1-3'UTR plants (Fig. 5A,B), unclosed petal tubes (Fig. 5C), severely wrinkled petals, and/or stigmas in one stamen were observed in the TRV2::NbGLO2-3'UTR flowers (Fig. $5 D, E$ ). The qPCR analysis showed that the expressions of the target genes were significantly downregulated in both cases (Fig. 5F, $G$ and Table 3 Suppl.). In these analyses, four categories of flowers (N0 - N3) were selected from
TRV2::NbGLO2-3'UTR (Fig. 5G), and four TRV2::NbGLO1-3'UTR flower categories demonstrated different degrees of gene silencing (Fig. $5 F$ ) although no phenotypic variations were observed. Meanwhile, the expression of $N b A P 1$ was also upregulated in the both cases (Fig. $5 F, G$ ). These results suggest that gene silencing triggered by the 3'UTR was effective and gene-specific. However, the extent of phenotypic variation when only the 3'UTR was used as a triggered sequence was indeed weaker than previous observations, in which part of the coding sequences were included in the trigger constructs (Geuten and Irish 2010). The insufficient downregulation of another paralog triggered by the 3'UTR fragments might account for the observed weak to negligible phenotypic variation, further indicating that a single $\mathrm{NbGLO1}$ or $\mathrm{NbGLO2}$ mutant was unlikely to be capable of inducing pronounced floral variations. 
To assess variations in gene silencing using different trigger sequences, we conducted sequence analysis of the target gene sequence and the trigger sequence. The $N b P D S$ and the 409-bp trigger $V p P D S$ fragment showed $79.7 \%$ nucleotide identity, whereas a 26-nucleotide stretch depicted $100 \%$ identity (Fig. 3 Suppl.). The VpPI-ORF trigger sequence was $356 \mathrm{bp}$ long and shared 65.6 and $69.0 \%$ identity at the nucleotide level with the putative

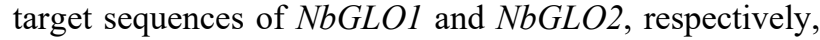
and there was a 23-nucleotide stretch in $\mathrm{VPPI}$ and $\mathrm{NbGLOI}$ or $N b G L O 2$ showing $100 \%$ identity, with one base mismatch (Table 1 and Fig. 3 Suppl.). The VpPI-3'UTR trigger sequence was $177 \mathrm{bp}$ long and shared 45.6 and $51.8 \%$ sequence identity at the nucleotide level with those of $N b G L O 1-3$ 'UTR and $N b G L O 2-3$ 'UTR, respectively, and the number of mismatched bases was 8 and 7 , respectively, within a 21-nucleotide stretch shared between the $V p P I-3$ 'UTR sequence and the NbGLO1-3'UTR, and NbGLO2-3'UTR sequences (Table 1 and Fig. 3 Suppl.). The trigger sequences of both $V p P D S$ ORF and VPPI-ORF imparted strong gene silencing effects, which might be due to the high sequence identity between the exogenous genes and the trigger sequence and the longer length of the trigger sequences (Table 1 and Table 2 Suppl.). These analyses indicate that sequence identity was not the only determinant for effective gene silencing. Nonetheless, a pure 3'UTR was effective in inducing specific gene silencing, as expected.
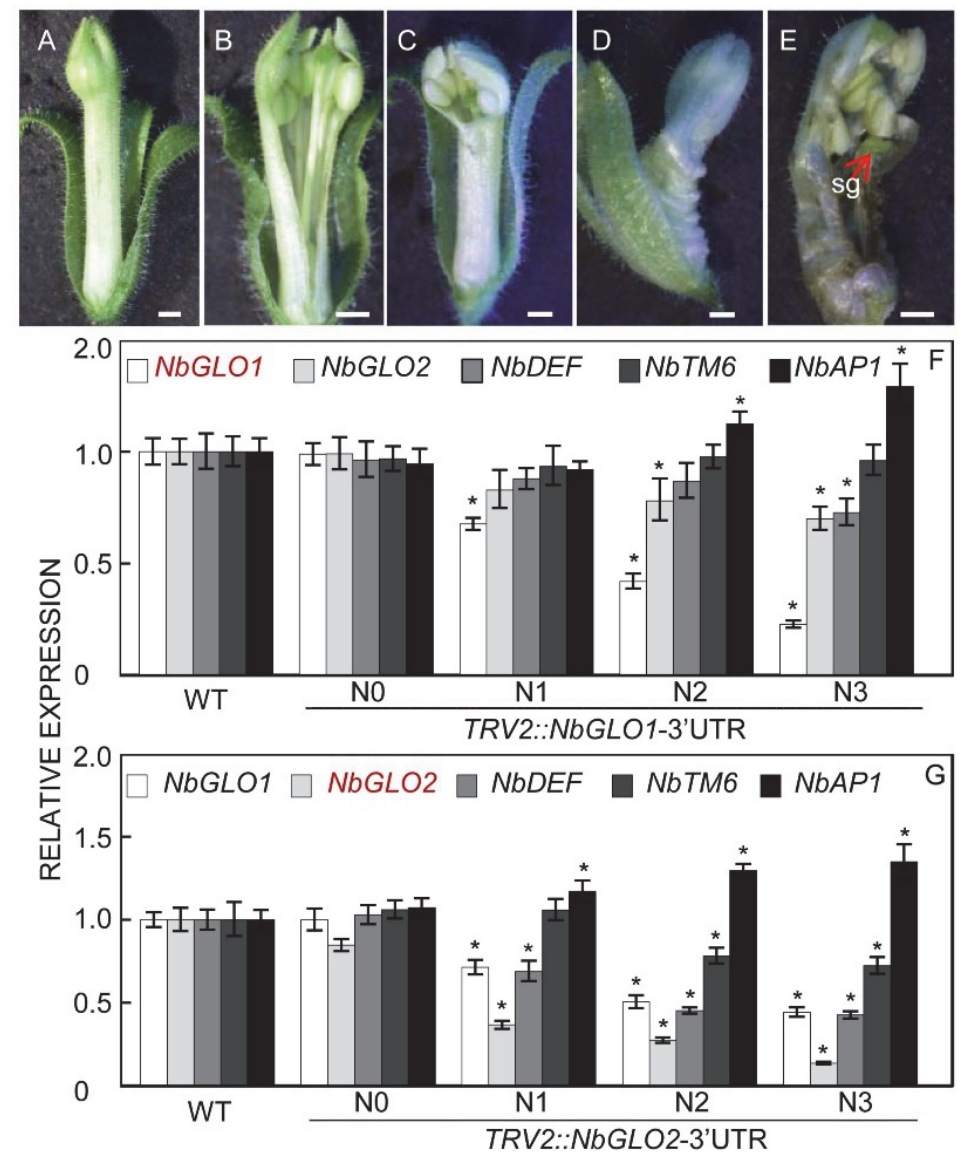

Fig. 5. Silencing $N b G L O$ by the 3 'UTR sequences of the $N b G L O$ genes. $A$ - A wild-type flower. $B$ - A wild-type flower of a $T R V 2: \because N b G L O 1-3$ 'UTR-inoculated plant. $C$ - A flower of a TRV2::NbGLO2-3'UTR virus-induced gene silencing (VIGS) plant with unclosed petal tubes. $D$ - A flower of a TRV2::NbGLO2-3'UTR VIGS plant with wrinkled petals. $E$ - A flower of a TRV2::NbGLO23'UTR VIGS plant with curving filaments and a stamen that transformed into a stigma-like structure. $F$ - Relative expressions of B-class MADS-box genes and NbAP1 in VIGS flowers as mediated by TRV2::NbGLO1-3'UTR. WT - wild-type flowers; N0 to N3 -

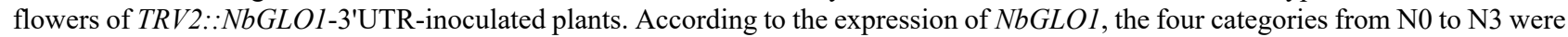
selected. $G$ - Relative expressions of B-class genes and $N b A P 1$ in VIGS flowers mediated by TRV2::NbGLO2-3'UTR. WT - wild-type flowers; N0 - WT-like flowers in the TRV2::NbGLO2-3'UTR VIGS plants. N1 - flowers with unclosed petal tubes; N2 - flowers with crumpled corolla tubes; N3 - flowers with crumpled corolla tubes and one anther that was transformed into a stigma-like structure. Means \pm SDs, $n=3, *$-significant differences at $P \leq 0.01$ relative to WT. The flowers were collected about 60 d post-inoculation. 


\section{Discussion}

Virus-induced gene silencing is a particularly useful tool for plant functional genomics because it permits the knock down of genes of interest, the observation of elicited phenotypes within one month, and does not require the creation of stable plant lines (Burch-Smith et al. 2004). Efficient VIGS has been successfully accomplished in a few plant species, and more than 30 VIGS vectors have been developed, with the majority having a narrow host range (Yuan et al. 2011). Tobacco rattle virus is a VIGS system that was initially designed for Solanaceaous plants, with wide applications to other plant species (Gould and Kramer 2007, Tian et al. 2015, Bilichak and Kovalchuk
2017). The present study first tried to apply the system to $V$. philippica, an optimal model for investigating the developmental evolution of dimorphic flowers ( $\mathrm{Li}$ et al. 2016). The attempt was not successful because no systemic gene silencing effect was observed in $V$. philippica. This might be due to the limited distribution of TRV transcripts in this Viola species, as observed in Eschscholzia californica (Wege et al. 2007). Nonetheless, we observed the systemic gene silencing effect in $N$. benthamiana when both the $N$. benthamiana-native and $V$. philippica-derived gene probes were used, which indicates that heterologous probes effectively triggered VIGS.

Table 1. Nucleotide sequence identities and mismatches between trigger and target sequences. For details, see Table 3 Suppl.

\begin{tabular}{|c|c|c|c|c|c|c|c|c|c|}
\hline $\begin{array}{l}\text { Trigger } \\
\text { sequence }\end{array}$ & $\begin{array}{l}\text { Gene } \\
\text { silencing } \\
\text { extent } \\
\mathrm{NbGLO1} \\
{[\%]}\end{array}$ & $\begin{array}{l}\text { Gene } \\
\text { silencing } \\
\text { extent } \\
\mathrm{NbGLO2} \\
{[\%]}\end{array}$ & $\begin{array}{l}\text { Inserted } \\
\text { cDNA } \\
\text { length } \\
\text { [bp] }\end{array}$ & $\begin{array}{l}\text { Nucleotide } \\
\text { identity } \\
\text { with } \\
\mathrm{NbGLOI} \\
{[\%]}\end{array}$ & $\begin{array}{l}\text { Nucleotide } \\
\text { identity } \\
\text { with } \\
N b G L O 2 \\
{[\%]}\end{array}$ & $\begin{array}{l}\text { Maximum } \\
\text { stretch of } \\
\text { nucleotides } \\
\text { having } \\
100 \% \\
\text { identity } \\
\text { with } \\
\mathrm{NbGLO1}\end{array}$ & $\begin{array}{l}\text { Maximum } \\
\text { stretch of } \\
\text { nucleotides } \\
\text { having } \\
100 \% \\
\text { identity } \\
\text { with } \\
\mathrm{NbGLO2}\end{array}$ & $\begin{array}{l}\text { Number of } \\
\text { nucleotide } \\
\text { mismatch in } \\
21 \text {-nucleotide } \\
\text { stretch having } \\
10 \% \text { identity } \\
\text { with } N b G L O 1\end{array}$ & $\begin{array}{l}\text { Number of } \\
\text { nucleotide } \\
\text { mismatch in } \\
\text { 21-nucleotide } \\
\text { stretch having } \\
100 \% \\
\text { identity with } \\
\mathrm{NbGLO2}\end{array}$ \\
\hline $\begin{array}{l}\text { VpPI } \\
\text { ORF }\end{array}$ & 64.00 & 70.74 & 356 & 65.6 & 69.0 & 13 & 19 & 1 & 1 \\
\hline $\begin{array}{l}\text { VpPI } \\
\text { 3'UTR }\end{array}$ & 46.70 & 68.68 & 177 & 45.6 & 51.8 & 6 & 5 & 8 & 7 \\
\hline $\begin{array}{l}\text { NbGLOI } \\
\text { 3'UTR }\end{array}$ & 55.86 & 22.97 & 161 & 100.0 & 39.9 & 161 & 3 & 0 & 11 \\
\hline $\begin{array}{l}\mathrm{NbGLO2} \\
\text { 3'UTR }\end{array}$ & 44.61 & 74.11 & 181 & 39.9 & 100.0 & 3 & 181 & 11 & 0 \\
\hline
\end{tabular}

This phenomenon may easily occur between closely related species; for example, gene probes from one Solanaceaeous species could silence the corresponding genes in the other Solanaceaeous species (Ryu et al. 2004, Senthil-Kumar et al. 2007) although a dsRNA fragment (a silencing inducer) of $300-500 \mathrm{bp}$ in length with at least one stretch of 23 nucleotides with a $100 \%$ identity to a targeted transgene mRNA is essential (Thomas et al. 2001). Our work has added further evidence that gene silencing occurs in $N$. benthamiana using gene probes from distantly related species (Howes and Kumagai 2005, Senthil-Kumar et al. 2006). Furthermore, our findings also indicate that a $100 \%$ nucleotide sequence identity between the target gene and the probe is not essential for VIGS. Nevertheless, there is a need to determine the minimal nucleotide identity and number of base mismatches between the trigger sequence and the target sequence that could effectively trigger a knockdown effect on endogenous genes. We took advantage of the probe construct that we made using genes from $V$. philippica to test this in $N$. benthamiana.

The $P D S$ gene is a structural gene that is conserved among plant species and is often used as a reporter for gene silencing (Chen et al. 2004, Burch-Smith et al. 2006, Yuan et al. 2011, Liu et al. 2014). The VpPDS has a $79.7 \%$ nucleotide identity and a 26-nucleotide stretch that shares $100 \%$ identity with $\mathrm{NbPDS}$; therefore, $100 \%$ gene silencing was observed. However, different situations were observed when $V p P I$ was used as a probe. The $V p P I$ gene encodes a B-class MADS-box regulatory protein in $V$. philippica (Li et al. 2016) and is the putative ortholog of $N b G L O 2$. We considered only the silencing effect of the orthologous gene pair, and other downregulations are considered to be reciprocal regulations among B-class MADS-box genes in Nicotiana (Geuten and Irish 2010). The VpPI-ORF fragment that was used as a trigger sequence had a $69 \%$ nucleotide identity with the corresponding target section of $\mathrm{NbGLO2-ORF}$ in Nicotiana. Except for the single-base mismatch, a 21-nucleotide stretch with a $100 \%$ identity between $\mathrm{VpPI-ORF}$ and $\mathrm{NbGLO2-ORF}$ was detected. $N b G L O 2$ was effectively knocked down, which induced visible floral phenotypic variations, wherein the pistils replaced stamens, and the petals shrank. The wrinkled petals 
seemed to be the consequence of the strong transformation of petal midveins into sepal midveins, thereby forcing the second whorl floral organ to fold (Geuten and Irish 2010). Therefore, VpPI in Viola plays the same role as its ortholog NbGLO2 in Nicotiana. In this case, the number of base mismatches coincides with previous findings (SenthilKumar et al. 2007). However, the 3'UTR sequence of VpPI had only a $51.8 \%$ nucleotide identity with the corresponding sequence in $\mathrm{NbGLO2}$, and up to 7 base mismatches within a 21-nucleotide stretch compared to the target sequence of $\mathrm{NbGLO2-3'UTR}$ were observed. NbGLO2 was also efficiently knocked down in $N$. benthamiana, which in turn induced floral phenotypic variations. Nonetheless, no $\mathrm{NbGLO1}$ and $\mathrm{NbGLO2}$ were silenced in the TRV2::VpPDS inoculated flowers, no $N b A P 1$ was silenced in the flowers inoculated with TRV2::VpPI-3'UTR constructs, and no mutated flowers were observed in plants inoculated with the TRV2::NbGLO1-3'UTR constructs. These observations further support that the silencing phenotypes in flowers with TRV2::VpPI-3'UTR constructs are specific for the $V p P I-3$ 'UTR construct in $N$. benthamiana instead of the side effects of silencing process. Thus, a 21-nucleotide stretch of identity with $1-7$ base mismatches was sufficient for silencing target genes in this particular case. Moreover, the native trigger sequences NbGLO2-3'UTR and $N b G L O 1-3$ 'UTR demonstrated different degrees of gene silencing. However, the specificity and mechanism of gene silencing triggered by the lower homology sequence need investigations. We currently support that the length and position of the triggers in the cDNA may affect the efficiency of silencing target genes (Liu and Page 2008), and the structure of RNA might also play a putative role (Senthil-Kumar et al. 2007). In addition, using the 3'UTR as a trigger sequence for effective gene silencing did not lead to the same phenotypic deviations as those observed using ORFs (Geuten and Irish 2010) and heterologous VpPI-ORF fragments (this study), where both paralogs were significantly downregulated, thereby indicating that

\section{References}

Becker, A., Lange, M.: VIGS-genomics goes functional. - Trends Plant Sci. 15: 1-4, 2010.

Benavente, L.M., Ding, X.S., Redinbaugh, M.G., Nelson, R.S., Balint-Kurti, P.: Virus-induced gene silencing in diverse maize lines using the brome mosaic virus-based silencing vector. - Maydica. 57: 206-214, 2012.

Bilichak, A., Kovalchuk, I.: Increasing a stable transformation efficiency of Arabidopsis by manipulating the endogenous gene expression using virus-induced gene silencing. Methods mol. Biol. 1456: 225-236, 2017.

Bowman, J.L., Smyth, D.R., Meyerowitz, E.M.: Genes directing flower development in Arabidopsis. - Plant Cell 1: 37-52, 1989.

Brigneti, G., Martin-Hernandez, A.M., Jin, H., Chen, J., Baulcombe, D.C., Baker, B., Jones, J.D.: Virus-induced gene the two paralogs $N b G L O 1$ and $N b G L O 2$ might play similar roles in organ identity determination during stamens and corolla development.

Homologous genes, including orthologous and paralogous genes, generally share similar functions or functions in closely related pathways (Vandenbussche et al. 2003, Geuten and Irish 2010, Roque et al. 2013, Zhang et al. 2015). TRV-mediated gene silencing technology is currently not applicable to $V$. philippica; however, the findings of the present study provide a platform for inferring the function of specific plant genes where a native functional inference is impossible. For example, in $V$. philippica, $V p P I$ could knock $N b G L O 2$ down and induce phenotypic variations similar to the NbGLO2 mutant (Geuten and Irish 2010), thereby suggesting that VpPI functions as a B-class MADS-box gene, which determines petal and stamen organ identities

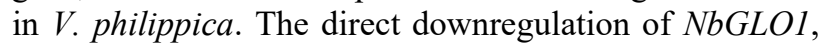

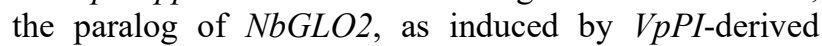
triggers, is not excluded although the reciprocal regulation among B-class MADS-box genes has been observed (Geuten and Irish 2010, Zhang et al. 2014b, Zhang et al. 2015). However, the phenotype of the mutated flowers with the $V p P I-3$ 'UTR as a trigger sequence was similar to that using NbGLO2-3'UTR as a trigger sequence. VpPI-3'UTR should be a specific probe, which is similar to NbGLO2-3'UTR. Therefore, designing gene-specific probes for gene silencing or knockdowns should be performed with caution. If trigger sequences are not specific, then all paralogous genes, instead of a single gene, could be knocked down, and the observed phenotypic variations might be the collective effect of silencing all paralogous genes instead of a single paralogous gene, thereby resulting in unreliable functional inferences. Therefore, when no relative gene-specific coding region encompassing the variable region of the gene of interest is available, the 3'UTR may be included in the selection of trigger sequences.

silencing in Solanum species. - Plant J. 39: 264-272, 2004.

Burch-Smith, T.M., Anderson, J.C., Martin, G.B., DineshKumar, S.P.: Applications and advantages of virus-induced gene silencing for gene function studies in plants. - Plant J. 39: 734-746, 2004.

Burch-Smith, T.M., Schiff, M., Liu, Y., Dinesh-Kumar, S.P.: Efficient virus-induced gene silencing in Arabidopsis. - Plant Physiol. 142: 21-27, 2006.

Chen, J.C., Jiang, C.Z., Gookin, T.E., Hunter, D.A., Clark, D.G., Reid, M.S.: Chalcone synthase as a reporter in virus-induced gene silencing studies of flower senescence. - Plant mol. Biol. 55: 521-530, 2004.

Chung, E., Seong, E., Kim, Y.C., Chung, E.J., Oh, S.K., Lee, S., Park, J.M., Joung, Y.H., Choi, D.: A method of high frequency virus-induced gene silencing in chili pepper 
(Capsicum annuum L. cv. Bukang). - Mol. Cells 17: 377-380, 2004.

Constantin, G.D., Krath, B.N., MacFarlane, S.A., Nicolaisen, M., Johansen, I.E., Lund, O.S.: Virus-induced gene silencing as a tool for functional genomics in a legume species. - Plant J. 40: 622-631, 2004.

Dinesh-Kumar, S.P., Anandalakshmi, R., Marathe, R., Schiff, M., Liu, Y.: Virus-induced gene silencing. - Methods mol. Biol. 236: 287-294, 2003.

Gao, X., Britt, R.C., Jr., Shan, L., He, P.: Agrobacteriummediated virus-induced gene silencing assay in cotton. - J. Vis. Exp. 54: 2938, 2011.

Geuten, K., Irish, V.: Hidden variability of floral homeotic B genes in Solanaceae provides a molecular basis for the evolution of novel functions. - Plant Cell 22: 2562-2578, 2010.

Gould, B., Kramer, E.M.: Virus-induced gene silencing as a tool for functional analyses in the emerging model plant Aquilegia (columbine, Ranunculaceae). - Plant Methods 3: 6, 2007.

Hein, I., Barciszewska-Pacak, M., Hrubikova, K., Williamson, S., Dinesen, M., Soenderby, I.E., Sundar, S., Jarmolowski, A., Shirasu, K., Lacomme, C.: Virus-induced gene silencingbased functional characterization of genes associated with powdery mildew resistance in barley. - Plant Physiol. 138: 2155-2164, 2005.

Hill, J.P., Lord, E.M.: Floral development in Arabidopsis thaliana: a comparison of the wild type and the homeotic pistillata mutant. - Can. J. Bot. 67: 2922-2936, 1989.

Howes, T., Kumagai, M.H.: Virus-induced gene silencing in Nicotiana benthamiana using DEAD-box helices sequence derived from Dunaliella salina. - J. Young Investig. 12: 1-6, 2005.

Kumagai, M.H., Donson, J., Della-Cioppa, G., Harvey, D., Hanley, K., Grill, L.K.: Cytoplasmic inhibition of carotenoid biosynthesis with virus-derived RNA. - Proc. nat. Acad. Sci. USA 92: 1679-1683, 1995.

Li, Q.X., Huo, Q.D., Wang, J., Zhao, J., Sun, K., He, C.Y.: Expression of B-class MADS-box genes in response to variations in photoperiod is associated with chasmogamous and cleistogamous flower development in Viola philippica. BMC Plant Biol. 6: 151, 2016.

Liu, E., Page, J.E.: Optimized cDNA libraries for virus-induced gene silencing (VIGS) using tobacco rattle virus. - Plant Methods 4: 5, 2008.

Liu, Y., Schiff, M., Dinesh-Kumar, S.P.: Virus-induced gene silencing in tomato. - Planta 31: 777-786, 2002.

Liu, Y., Sun, W., Zeng, S., Huang, W., Liu, D., Hua, W., Shen, X., Wang, Y.: Virus-induced gene silencing in two novel functional plants, Lycium barbarum L. and Lycium ruthenicum Murr. - Sci. horticult. 170: 267-274, 2014.

Livak, K.J., Schmittgen, T.D.: Analysis of relative gene expression data using real-time quantitative PCR and the $2^{-}$ $\triangle \triangle \mathrm{CT}$ method. - Methods 25: 402-408, 2001.

Ma, M., Yan, Y., Huang, L., Chen, M., Zhao, H.: Virus-induced gene-silencing in wheat spikes and grains and its application in functional analysis of HMW-GS-encoding genes. - BMC Plant Biol. 12: 141, 2012.

Meng, L.H., Wang, R.H., Zhu, B.Z., Zhu, H.L., Luo, Y.B., Fu, D.Q.: Efficient virus-induced gene silencing in Solanum rostratum. - PLoS ONE 11: e0156228, 2016.

Pflieger, S., Blanchet, S., Camborde, L., Drugeon, G., Rousseau, A., Noizet, M., Planchais, S., Jupin, I.: Efficient virus-induced gene silencing in Arabidopsis using a 'onestep’ TYMV-derived vector. - Plant J. 56: 678-690, 2008.

Purkayastha, A., Mathur, S., Verma, V., Sharma, S., Dasgupta, I.: Virus-induced gene silencing in rice using a vector derived from a DNA virus. - Planta 232: 1531-1540, 2010.

Ratcliff, F., Harrison, B.D., Baulcombe, D.C.: A similarity between viral defense and gene silencing in plants. - Science 276: 1558-1560, 1997.

Ratcliff, F., Martin-Hernandez, A.M., Baulcombe, D.C.: Tobacco rattle virus as a vector for analysis of gene function by silencing. - Plant J. 25: 237-245, 2001.

Robertson, D.: VIGS vectors for gene silencing: many targets, many tools. - Annu. Rev. Plant Biol. 55: 495-519, 2004.

Roque, E., Serwatowska, J., Rochina, M.C., Wen, J.Q., Mysore, K.S., Yenush, L., Beltrán, J.P., Caňas, L.A.: Functional specialization of duplicated AP3-like genes in Medicago truncatula. - Plant J. 73: 663-675, 2013.

Ryu, C.M., Anand, A., Lang, L., Mysore, K.S.: Agrodrench: a novel and effective agroinoculation method for virus-induced gene silencing in roots and diverse Solanaceous species. Plant J. 40: 322-331, 2004.

Sasaki, S., Yamagishi, N., Yoshikawa, N.: Efficient virus-induced gene silencing in apple, pear and Japanese pear using apple latent pherical virus vectors. - Plant Methods 7: 15, 2011.

Senthil-Kumar, M., Hema, R., Anand, A., Kang, L., Udayakumar, M., Mysore, K.S.: A systematic study to determine the extent of gene silencing in Nicotiana benthamiana and other Solanaceae species when heterologous gene sequences are used for virus-induced gene silencing. - New Phytol. 176: 782-791, 2007.

Senthil-Kumar, M., Udayakumar, M.: High-throughput virusinduced gene-silencing approach to assess the functional relevance of a moisture stress-induced cDNA homologous to lea4. - J. exp. Bot. 57: 2291-2302, 2006.

Sundström, J. F., Nakayama, N., Glimelius, K., Irish, V.F.: Direct regulation of the floral homeotic APETALA1 gene by APETALA3 and PISTILLATA in Arabidopsis. - Plant J. 46: 593-600, 2006.

Tamura, K., Peterson, D., Peterson, N., Stecher, G., Nei, M., Kumar, S.: MEGA5: molecular evolutionary genetics analysis using maximum likelihood, evolutionary distance, and maximum parsimony methods. - Mol. Biol. Evol. 28: 2731-2739, 2011.

Thomas, C.L., Jones, L., Baulcombe, D.C., Maule, A.J.: Size constraints for targeting post transcriptional gene silencing and for RNA-directed methylation in Nicotiana benthamiana using a potato virus X vector. - Plant J. 25: 417-425, 2001.

Tian, J., Cheng, L., Han, Z., Yao, Y.: Tobacco rattle virus mediated gene silencing in strawberry plants. - Plant Cell Tissue Organ Cult. 120: 1131-1138, 2015.

Tuttle, J. R., Haigler, C.H., Robertson, D.: Method: low-cost delivery of the cotton leaf crumple virus-induced gene silencing system. - Plant Methods 8: 27, 2012.

Vandenbussche, M., Theissen, G., Van de Peer, Y., Gerats, T.: Structural diversification and neo-functionalization during floral MADS-box gene evolution by C-terminal frame shift mutations. - Nucl. Acids Res. 31: 4401-4409, 2003.

Wege, S., Scholz, A., Gleissberg, S., Becker, A.: Highly efficient virus-induced gene silencing (VIGS) in California poppy (Eschscholzia californica): an evaluation of VIGS as a strategy to obtain functional data from non-model plants. Ann. Bot. 100: 641-649, 2007.

Yuan, C., Li, C., Yan, L., Jackson, A.O., Liu, Z., Han, C., Yu, J., 
Li, D.: A high throughput barley stripe mosaic virus vector for virus induced gene silencing in monocots and dicots. PLoS ONE 6: e26468, 2011.

Zahn, L.M., Leebens-Mack, J., De Pamphilis, C.W., Ma, H., Theissen, G.: To be or not to be a flower: the role of DEFICIENS and GLOBOSA orthologs in the evolution of the angiosperms. - J. Hered. 96: 225-240, 2005.

Zhang, C., Bradshaw, J.D., Whitham, S.A., Hill, J.H.: The development of an efficient multipurpose bean pod mottle virus viral vector set for foreign gene expression and RNA silencing. - Plant Physiol. 153: 52-65, 2010.

Zhang, C., Ghabrial, S.A.: Development of bean pod mottle virus-based vectors for stable protein expression and sequence-specific virus-induced gene silencing in soybean. -
Virology 344: 401-411, 2006.

Zhang, J.S., Li, Z.C., Zhao, J., Zhang, S.H., Quan, H., Zhao, M., He, C.Y.: Deciphering the Physalis floridana doublelayered-lantern 1 mutant provides insights into functional divergence of the GLOBOSA duplicates within the Solanaceae. - Plant Physiol. 164: 748-764, 2014a.

Zhang, J.S., Zhao, J., Zhang, S.H., He, C.Y.: Efficient gene silencing mediated by tobacco rattle virus in an emerging model plant Physalis. - PLoS ONE 9: e85534, 2014b.

Zhang, S.H., Zhang, J.S., Zhao, J., He, C.Y.: Distinct subfunctionalization and neofunctionalization of the B-class MADS-box genes in Physalis floridana. - Planta 241: 387402, 2015. 